\title{
Fatores de risco relacionados à obesidade em escolares atendidos em um ambulatório de pediatria
}

\author{
Risk factors associated with obesity in gradeschoolers attended in a pediatric clinic
}

Factores de riesgo relacionados a la obesidad en escolares atendidos en un ambulatorio de pediatría

Ana Plycila Botelho Chaves ${ }^{1,2}$, Ana Luiza Lopes de Freitas Freire ${ }^{1 *}$, Dilma Costa de Oliveira Neves $^{1,3}$, Kátia Soares de Oliveira ${ }^{1,3,4,5}$, Marina Lopes de Freitas Freire ${ }^{6}$.

\section{RESUMO}

Objetivo: Identificar fatores de risco relacionados à obesidade em escolares atendidos em um ambulatório de pediatria e correlacioná-los. Métodos: Trata-se de um estudo observacional, transversal, com coleta sistematizada de dados acerca da prática de atividade física e níveis pressóricos dos escolares, além de dados antropométricos dos escolares e seus pais em um ambulatório de pediatria, no período de setembro a dezembro de 2015. Foram incluídas crianças na faixa etária escolar, de 5 a 12 anos exclusive de idade. Resultados: Observou-se uma prevalência de 38\% de sobrepeso e 16\% de obesidade. Quanto aos níveis pressóricos, $34 \%$ estavam dentro do percentil $50,38 \%$ no percentil $90,16 \%$ no percentil 95 e $12 \%$ no percentil 99 de pressão arterial. Quanto à atividade física, $86 \%$ foram classificados como ativos e $14 \%$ como inadequadamente ativos. Quanto à regularidade da prática da atividade física, 30\% praticavam atividade física regularmente e $70 \%$ de forma irregular. Conclusão: A prevalência de obesidade infantil foi de $16 \%$ e $38 \%$ de sobrepeso. Não houve relação de obesidade com práticas de atividade física e IMC dos pais. Foi forte a relação entre obesidade e níveis pressóricos aumentados. Modificações do estilo de vida para prevenção e tratamento da obesidade são de grande importância para prevenir eventos cardiovasculares no futuro.

Palavras-chave: Obesidade infantil, Fatores de risco, Pediatria.

\begin{abstract}
Objective: To identify risk factors associated with obesity in gradeschoolers attended in a pediatric clinic and correlate them. Methods: This is a transversal and observational study with collection of data on physical activity, blood pressure levels of gradeschoolers and anthropometric data of the gradeschoolers and their parents in a pediatric clinic from september to december 2015. Children were included in the gradeschooler age from 5 to 12 years old. Results: There was a prevalence of $38 \%$ of overweight and $16 \%$ of obesity. About the blood pressure levels, $34 \%$ were at 50th percentile, $38 \%$ at 90 th percentile, $16 \%$ at 95 th percentile and $12 \%$ at 99th percentile of blood pressure. $86 \%$ were classified as active and $14 \%$ as inappropriately active. About the regular practice of physical activity, 30\% practiced regular physical activity and $70 \%$ practiced irregularly. Conclusion: The prevalence of childhood obesity was $16 \%$ and $38 \%$ of overweight. There was no association between obesity, physical activity practices and BMI of the parents. However, it was found a strong relationship between obesity and increased blood pressure levels. The lifestyle changes to prevention and treatment of obesity are really important in preventing cardiovascular events in the future.
\end{abstract}

Key words: Childhood obesity, Risk factors, Pediatrics.

${ }^{1}$ Centro Universitário do Estado do Pará. Belém - PA.

${ }^{2}$ Hospital Materno Infantil de Goiânia. Goiânia - GO. *E-mail: analuiza freire@hotmail.com

3Universidade Federal do Pará. Belém - PA.

${ }^{4}$ Santa Casa de Misericórdia do Pará. Belém - PA.

5Universidade do Estado de São Paulo. Botucatu - SP.

${ }^{6}$ Faculdade Metropolitana da Amazônia. Belém - PA. 


\section{RESUMEN}

Objetivo: Identificar factores de riesgo relacionados a la obesidad en escolares atendidos en un ambulatorio de pediatría y correlacionarlos. Métodos: Se trata de un estudio observacional, transversal, con colecta de datos acerca de la práctica de actividad física y niveles presóricos de los escolares, datos antropométricos de los escolares y sus padres en un ambulatorio de pediatría, en el período de septiembre a diciembre de 2015. Se incluyeron niños en el grupo de edad escolar, de 5 a 12 años de edad. Resultados: Se observó una prevalencia del $38 \%$ de sobrepeso y $16 \%$ de obesidad. $34 \%$ estaban dentro del percentil 50 , $38 \%$ en el percentil $90,16 \%$ en el percentil 95 y $12 \%$ en el percentil 99 de presión arterial. $86 \%$ fue clasificado como activos y $14 \%$ como inadecuadamente activos. $30 \%$ practicaba actividad física regularmente y $70 \%$ de forma irregular. Conclusión: La prevalencia de obesidad infantil fue de $16 \%$ y $38 \%$ de sobrepeso. No hubo relación de obesidad con prácticas de actividad física e IMC de los padres. Fue fuerte la relación entre obesidad y niveles presóricos aumentados. Las modificaciones del estilo de vida para la prevención y el tratamiento de la obesidad son de gran importancia para prevenir eventos cardiovasculares en el futuro.

Palabras clave: Obesidad infantil, Factores de riesgo, Pediatría.

\section{INTRODUÇÃO}

A obesidade, considerada uma epidemia mundial, pode ser caracterizada pelo acúmulo excessivo de tecido adiposo no organismo, oriunda de causa multifatorial, como suscetibilidade genética, sexo, idade, ocupação, dieta e outros. Tal patologia pode nem sempre estar ligada a doenças endocrinológicas. O tipo de obesidade que se encaixa nesse perfil geralmente tem forte relação com o desbalanço alimentar resultante do desequilíbrio entre a ingestão e a necessidade diária de nutrientes para suprir o metabolismo de um indivíduo geneticamente suscetível (PASCHOAL et al., 2009).

O panorama mundial e brasileiro da obesidade tem se revelado como um novo desafio para a saúde pública, uma vez que sua incidência e prevalência têm crescido de forma alarmante nos últimos 30 anos. No Brasil, a mudança no perfil nutricional está ganhando grande importância como um modelo de atenção à saúde, incorporando ações de promoção e prevenção da saúde e tratamento da obesidade e de doenças crônicas não transmissíveis (REIS et al., 2011).

$\mathrm{Na}$ infância e adolescência, a obesidade atinge diversas idades, sexos, etnias e rendas familiares (TENORIO e COBAYASHI, 2011). Ainda segundo Tenorio e Cobayashi (2011), estudos realizados nos Estados Unidos, entre 1999 e 2003, comprovam um aumento na prevalência de sobrepeso em crianças e adolescentes.

No Brasil, segundo o último estudo antropométrico realizado pelo IBGE na faixa etária de 10 a 19 anos de idade, entre 2008 e 2009 , a prevalência de excesso de peso foi de $21,5 \%$ nos meninos e $19,4 \%$ nas meninas (BRASIL, 2010).

O aumento do consumo de alimentos com alto teor calórico e a diminuição da prática de atividade física contribuíram para o aumento da prevalência de sobrepeso e obesidade na população, especialmente em crianças e adolescentes, levando ao aparecimento de complicações precoces, como hipertensão arterial sistêmica, diabetes mellitus tipo 2, dislipidemia, apneia obstrutiva do sono e alguns tipos de câncer. Além disso, a obesidade tem um alto impacto nas doenças cardiovasculares, como a aterosclerose (CARDOSO et al., 2014).

A obesidade se destaca por ser simultaneamente uma doença e um fator de risco para outras doenças, como a hipertensão arterial sistêmica (HAS). Observa-se que o aparecimento de HAS está cada vez mais precoce entre crianças e adolescentes (BRASIL, 2006). O excesso de peso é um importante preditor da pressão arterial elevada em crianças. $O$ risco de pressão arterial elevada pode aumentar mais que o dobro a cada unidade aumentada de z-score do índice de massa corpórea (IMC). Assim, a classificação adequada do estado nutricional em crianças é um instrumento de alerta para o risco de hipertensão (MORAES et al., 2014). Níveis pressóricos elevados em crianças acarretam em HAS na vida adulta e contribuem para a ocorrência de eventos cardiovasculares (FRIEDEMANN et al., 2012; SUN et al., 2007). Atualmente, considera-se obrigatória 
a medida da pressão arterial (PA) a partir dos três anos de idade, anualmente, ou antes, disso, quando houver fatores de risco (NHBP, 2004; SILVA et al., 2007).

O excesso de peso na infância aumenta as chances de obesidade na idade adulta, além de constituir fator de risco para doenças cardiovasculares, dislipidemias, hipertensão arterial, entre outras (TENORIO e COBAYASHI, 2011; PEREIRA et al., 2009). Além disso, existem as implicações psicossociais, que podem se estender por toda a vida (TENORIO e COBAYASHI, 2011).

Atualmente, pesquisas tem se destacado ao demonstrar o envolvimento do acúmulo de gordura na região abdominal como fator de risco para doenças cardiovasculares e diabetes tipo 2. Paralelamente a isso, alguns autores afirmam que crianças que apresentam menor adiposidade abdominal são mais propensas a terem níveis de moderado a alto da capacidade funcional cardiorrespiratória, justificando a importância do exercício físico na regulação da diminuição da gordura abdominal e, por conseguinte, para a prevenção da diabetes tipo 2 e da obesidade total em crianças e adolescentes (PASCHOAL et al., 2009).

No entanto, a falta de envolvimento dos pais se torna um grande empecilho para a prevenção e tratamento da obesidade infantil, sendo considerada uma grande barreira pelos profissionais de saúde atuantes nessa área. Tenorio e Cobayashi (2011) sugerem que essa ausência de comprometimento dos pais tem como base a falta de percepção do peso real da criança e suas complicações na saúde em curto e em longo prazo.

Entender como os indivíduos interagem com o ambiente e como essa interação predispõe muitas crianças a adquirirem excesso de peso é a chave para o desenvolvimento de medidas preventivas no futuro (ROBINSON et al., 2015).

A obesidade está cada vez mais prevalente entre a população, acometendo crianças, adolescentes e adultos, aumentando sua prevalência proporcionalmente com a idade. Sendo assim, fez- se necessário realizar este estudo com o objetivo de identificar fatores de risco relacionados à obesidade em escolares atendidos em um ambulatório de pediatria.

\section{METODOLOGIA}

A pesquisa foi desenvolvida de acordo com os preceitos da Declaração de Nuremberg, declaração de Helsinki e resolução 466/2012 do Conselho Nacional de Saúde (BRASIL, 2013). Foi iniciada após aprovação do Comitê de Ética em Pesquisa em Seres Humanos sob o número do parecer 1.048.049 de 04/05/2015. O estudo foi realizado nas dependências do Centro de Especialidades Médicas do Centro Universitário do Estado do Pará (CEMEC- CESUPA), com os dados coletados após assinatura do TCLE pelos pais ou responsáveis e do Termo de Assentimento pelo escolar acima de 7 anos, considerando que a partir desta idade a criança já possui desenvolvimento cognitivo quanto ao registro de seu nome.

Trata-se de um estudo observacional, transversal, analítico, com coleta sistematizada de dados acerca de prática de atividade física e níveis pressóricos dos escolares, além de dados antropométricos (peso, altura e índice de massa corporal) dos escolares e respectivos pais em um ambulatório de pediatria. Abrangendo 50 crianças em idade escolar, de 5 a 12 anos exclusive de idade.

As variáveis do estudo foram agrupadas em sócio-demográficas (sexo, faixa etária), antropométricas (peso, altura e IMC), níveis pressóricos dos escolares e as variáveis preditoras de práticas de atividade física.

Os dados foram consolidados sob a forma de números absolutos e/ou proporções e a análise estatística foi executada com auxílio do programa BioEstat 5.3 e a diferença estatística significante aceita foi de valor alfa $<5 \%$ ( $p$-valor $<0,05)$. Para a análise estatística foi utilizado o teste qui-quadrado de aderência para proporções esperadas iguais a fim de detectar diferença significativa estatisticamente entre as proporções observadas. Para o estabelecimento de relação significativa entre fator de risco e o desfecho de interesse foi utilizado o teste $\mathrm{G}$ para amostras independentes. 


\section{RESULTADOS}

No período compreendido entre setembro e dezembro de 2015, foram coletados dados de 50 escolares.

Observou-se que 40\% (20) das crianças se encontravam eutróficas, 38\% (19) com sobrepeso e 16\% (8) obesas (Tabela 1). Não houve diferenças com significância estatística na distribuição dos escolares segundo IMC e faixa etária $(p=0,4070)$, sendo as diferenças observadas atribuídas ao acaso (Tabela 1).

Tabela 1 - Distribuição dos escolares, segundo o IMC e a faixa etária.

\begin{tabular}{lccccccc}
\hline & \multicolumn{5}{c}{ Faixa etária (anos) } & & \\
\cline { 2 - 5 } \multicolumn{1}{c}{ IMC } & $\mathbf{5} \mathbf{~ a ~}$ & $\%$ & $\mathbf{9}$ a $\mathbf{1 2}$ & $\%$ & Total & $\%$ \\
\hline Magreza & 1 & 33,3 & 2 & 66,7 & 3 & 6,0 \\
Eutrófico & 11 & 55,0 & 9 & 45,0 & 20 & 40,0 \\
Sobrepeso & 6 & 31,6 & 13 & 68,4 & 19 & 38,0 \\
Obesidade & 4 & 42,9 & 4 & 57,1 & 8 & 16,0 \\
\hline \multicolumn{1}{r}{ Total } & $\mathbf{2 2}$ & 44,0 & $\mathbf{2 8}$ & 56,0 & $\mathbf{5 0}$ & $\mathbf{1 0 0 , 0}$
\end{tabular}

Fonte: Protocolo de pesquisa, 2016. $p=0,4070$ (Teste do Qui-quadrado).

Houve uma maior prevalência dos percentis 34\% (50) e 38\% (90) de pressão arterial, no entanto $57,1 \%$ (4) dos obesos estavam inseridos no percentil 95 e $28,6 \%$ (3) deles no percentil 99 de PA. Após a aplicação do teste qui-quadrado pode-se observar com base na probabilidade do resultado do teste, que as diferenças apresentadas foram estatisticamente significativas $(p<0,05)$, essas diferenças não foram atribuídas ao acaso (Tabela 2).

Tabela 2 - Distribuição dos escolares, segundo o IMC e os percentis de pressão arterial.

\begin{tabular}{|c|c|c|c|c|c|c|c|c|c|c|}
\hline \multirow[b]{2}{*}{ IMC } & \multicolumn{8}{|c|}{ Percentis } & \multirow[b]{2}{*}{ Total } & \multirow[b]{2}{*}{$\%$} \\
\hline & P50 & $\%$ & P90 & $\%$ & P95 & $\%$ & P99 & $\%$ & & \\
\hline Magreza & 1 & 33,3 & 1 & 33,3 & 1 & 33,3 & 0 & 0,0 & 3 & 6,0 \\
\hline Eutrófico & 10 & 50,0 & 8 & 40,0 & 0 & 0,0 & 2 & 10,0 & 20 & 40,0 \\
\hline Sobrepeso & 6 & 31,6 & 9 & 47,4 & 3 & 15,8 & 1 & 5,3 & 19 & 38,0 \\
\hline Obesidade & 0 & 0,0 & 1 & 14,3 & 4 & 57,1 & 3 & 28,6 & 8 & 16,0 \\
\hline Total & 17 & 34,0 & 19 & 38,0 & 8 & 16,0 & 6 & 12,0 & 50 & 100,0 \\
\hline
\end{tabular}

Fonte: Protocolo de pesquisa, 2016. $p=0,0099$ (Teste do Qui-quadrado).

Observa-se que não há relação estatisticamente significativa $(p=0,5575)$ entre o índice de massa corporal do escolar e o do pai. Observa-se também a maior prevalência de pais com sobrepeso (42\%) com valor superior ao dos escolares (38\%). (Tabela 3) 
Tabela 3 - Distribuição dos escolares, segundo o IMC do escolar e IMC do pai.

\begin{tabular}{|c|c|c|c|c|c|c|c|}
\hline \multirow[b]{2}{*}{ IMC Criança } & \multicolumn{5}{|c|}{ IMC do Pai } & \multirow[b]{2}{*}{ Total } & \multirow[b]{2}{*}{$\%$} \\
\hline & Normal & Sobrepeso & $\begin{array}{c}\text { Obesidade } \\
\text { Grau } 1\end{array}$ & $\begin{array}{c}\text { Obesidade } \\
\text { Grau } 2\end{array}$ & $\begin{array}{c}\text { Obesidade } \\
\text { Grau } 3\end{array}$ & & \\
\hline Magreza & 1 & 2 & 0 & 0 & 0 & 3 & 6,0 \\
\hline Eutrófico & 5 & 8 & 6 & 0 & 1 & 20 & 40,0 \\
\hline Sobrepeso & 7 & 7 & 4 & 1 & 0 & 19 & 38,0 \\
\hline Obesidade & 1 & 4 & 2 & 1 & 0 & 8 & 16,0 \\
\hline Total & 14 & 21 & 12 & 2 & 1 & 50 & 100,0 \\
\hline$\%$ & 28,0 & 42,0 & 24,0 & 4,0 & 2,0 & 100,0 & \\
\hline
\end{tabular}

Fonte: Protocolo de pesquisa, 2016. $\mathrm{p}=0,5575$ (Teste do Qui-quadrado).

A prevalência de escolares obesos é de $16 \%$ enquanto que as mães têm a prevalência de $18 \%$. Não foi encontrada relação estatisticamente significativa $(p=0,4586)$ entre $o$ índice de massa corporal do escolar e 0 da mãe (Tabela 4).

Tabela 4 - Distribuição dos escolares, segundo o IMC do escolar e IMC da mãe.

\begin{tabular}{|c|c|c|c|c|c|c|c|}
\hline \multirow[b]{2}{*}{ IMC Criança } & \multicolumn{5}{|c|}{ IMC da Mãe } & \multirow[b]{2}{*}{ Total } & \multirow[b]{2}{*}{$\%$} \\
\hline & Normal & Sobrepeso & $\begin{array}{c}\text { Obesidade } \\
\text { Grau } 1\end{array}$ & $\begin{array}{c}\text { Obesidade } \\
\text { Grau } 2\end{array}$ & $\begin{array}{c}\text { Obesidade } \\
\text { Grau } 3\end{array}$ & & \\
\hline Magreza & 2 & 1 & 0 & 0 & 0 & 3 & 6,0 \\
\hline Eutrófico & 9 & 9 & 2 & 0 & 0 & 20 & 40,0 \\
\hline Sobrepeso & 5 & 10 & 2 & 1 & 1 & 19 & 38,0 \\
\hline Obesidade & 4 & 1 & 1 & 2 & 0 & 8 & 16,0 \\
\hline Total Geral & 20 & 21 & 5 & 3 & 1 & 50 & 100,0 \\
\hline$\%$ & 40,0 & 42,0 & 10,0 & 6,0 & 2,0 & 100,0 & \\
\hline
\end{tabular}

Fonte: Protocolo de pesquisa, 2016. $p=0,4586$ (Teste $G$ ).

Com relação à frequência da atividade física, 43 (86\%) foram classificados como ativos, entretanto $65,1 \%$ (28) não possuíam atividade física regular. Nenhum escolar foi classificado como inativo. (Tabela 5). 
Tabela 5 - Distribuição das crianças participantes do estudo segundo realização de atividade física.

\begin{tabular}{lcc}
\hline ATIVIDADE FísICA & $\mathrm{N}$ & $\%$ \\
\hline Inativo & 0 & 0,0 \\
Inadequadamente ativo & 7 & 14,0 \\
Ativo & $\mathbf{4 3}$ & $\mathbf{8 6 , 0}$ \\
--Com Atividade Física Regular & 15 & 34,9 \\
--Sem Atividade Física Regular & 28 & 65,1 \\
\hline Total & 50 & 100,0 \\
\hline
\end{tabular}

Fonte: Protocolo de pesquisa, 2016. $p<0,0001$ (Teste qui-quadrado de aderência).

\section{DISCUSSÃO}

A maioria dos escolares deste estudo encontrava-se eutrófica, entretanto, 38\% (19) foram classificados como sobrepeso e 16\% (8), como obesos (Tabela 1). O relatório da International Obesity Task Force (IOTF) de 2003, para a Organização Mundial da Saúde, estima que aproximadamente 10\% dos indivíduos entre 5 e 17 anos de idade apresentam excesso de gordura corporal, sendo que de 2 a $3 \%$ são obesos (SBP, 2012). Nos Estados Unidos, dados nacionais, de 1999-2000 estimam que 15,8\% das crianças de 6 a 11 anos de idade e 16,1\% dos adolescentes de 12 a 19 anos de idade apresentam IMC acima de percentil 95 (COOK et at., 2003). A prevalência de obesidade apresenta crescimento progressivo nos países em desenvolvimento por estarem "importando" determinados hábitos ocidentais. Apenas os países muito desfavorecidos economicamente parecem consistentemente protegidos da obesidade (OLIVEIRA et al., 2003).

Estudo afirma que a prevalência de obesidade é maior nos países desenvolvidos, quando comparada aos em desenvolvimento, relação alterada apenas pela cultura da população e acesso à informação e aos serviços de saúde (KINRA et al., 2000). Contudo, neste estudo, a prevalência de sobrepeso e obesidade foi superior, provavelmente devido ao tamanho da amostra utilizada no estudo.

Em Bogotá (Colômbia), um estudo com 118 crianças em uma Fundação Cardio-Infantil, encontrou excesso de peso em $28 \%$ das crianças (BRICEÑO et al., 2015).

No que se refere a dados nacionais, um estudo em uma Estratégia de Saúde da Família de Criciúma-SC avaliou 871 crianças de zero a dez anos, mostrando maior prevalência de crianças eutróficas $(73,4 \%)$, resultados esses semelhantes ao encontrado nesta pesquisa. Entretanto, nesse mesmo estudo, a prevalência de sobrepeso/obesidade foram inferiores $(8,2 \%$ e $2,5 \%$ respectivamente) às da nossa pesquisa (PEREIRA et al., 2012). Em um estudo realizado no município de Formiga - MG, em escolares da rede pública, a prevalência de obesidade encontrada $(11,48 \%$ ) foi maior que a de sobrepeso $(6,44 \%)$. (MELO et al., 2012).

Carvalho e colaboradores (2014) descreveu a situação nutricional de crianças menores de 5 anos moradoras de três cidades da região nordeste do Brasil. Foi encontrada uma maior proporção de excesso de peso do que déficit de peso. A prevalência de excesso de peso encontrada foi de 8,3\% em Barra de São Miguel - AL, 10,3\% em Cabedelo - PB e 5,9\% em Tibau do Sul - RN.

As doenças da vida moderna têm atingido crianças de forma preocupante. Obesidade já é vista como epidemia mundial, o que se acompanha de aumento de fatores de risco cardiovasculares, como a Hipertensão Arterial Sistêmica (HAS). (FRIEDEMANN et al., 2012)

Em Vila Velha (ES), foram avaliadas 817 crianças da rede pública, onde foi observada pressão arterial elevada em $7,3 \%$ das crianças. Foi identificada uma forte relação entre a presença de excesso de peso e a ocorrência de níveis pressóricos elevados nessa população (MORAES et al., 2014).

Em outro estudo realizado em uma clínica pediátrica privada, 80,1\% das crianças apresentavam níveis 
pressóricos normais. Foi observado que a maior parte das crianças eutróficas apresentou valores de PA normais e todas as crianças com sobrepeso ou obesidade tiveram valores pressóricos alterados (BORGES e SILVA, 2012). Identificando que o excesso de peso esteve associado à presença de pressão arterial elevada.

Burgos et al (2011) encontraram uma alta prevalência de sobrepeso e obesidade entre escolares de Santa Cruz do Sul - RS, que apresentaram níveis pressóricos superiores em comparação aos escolares de baixo peso e peso normal. O presente estudo identificou que $57,1 \%$ dos obesos estavam inseridos no percentil 95 e $28,6 \%$ deles no percentil 99 de PA $(p<0,05)$, demonstrando uma forte associação entre obesidade e valores pressóricos alterados (Tabela 2).

Sabe-se que crianças com os dois pais obesos apresentam maior risco de obesidade que aquelas com um ou nenhum dos pais obesos (LAKE et al., 1997).

Giugliano e Carneiro (2004) demonstraram que $74,33 \%$ dos pais das crianças com sobrepeso e obesidade apresentaram-se obesos, mostrando uma frequência de sobrepeso e obesidade mais elevada nos pais de crianças com sobrepeso ou obesidade quando comparado aos pais das crianças normais.

Em um estudo realizado com 329 crianças de 6 a 13 anos de idade e suas mães em Perth, Austrália Ocidental, identificou que ter uma mãe com excesso de peso aumenta a probabilidade de ser uma criança com sobrepeso e obesidade (GIBSON et al., 2007).

Em um estudo de coorte britânico realizado a partir de 1958, foi coletado peso e altura dos sujeitos do estudo aos 7,11,16, 23 e 33 anos. As medidas dos pais foram registradas quando as crianças tinham 11 anos. O estudo mostrou que em cada idade de acompanhamento, o IMC das crianças aumentava a medida que o IMC dos pais aumentava (LAKE et al., 1997). Observou-se, neste estudo, uma prevalência igual de pais e mães com sobrepeso (42\%) e maior que a prevalência de sobrepeso nos escolares (38\%). (Tabelas 3 e 4).

Na infância se desenvolvem os principais hábitos alimentares e de estilo de vida, com implicações no risco de doença cardíaca na idade adulta. Dieta rica em gorduras saturadas e pouca atividade física se correlacionam com obesidade, colesterol elevado e hipertensão arterial na infância, com predisposição à morte prematura por doença coronariana. A identificação e intervenção precoce nestes hábitos são importantes na prevenção das doenças cardiovasculares no adulto. Crianças com sobrepeso e pressão arterial elevada têm risco aumentado de obesidade e hipertensão na idade adulta (HINNIG et al., 2014).

Com relação à prática de atividades físicas, apesar de terem sido encontradas diferenças sem significância estatística, o atual estudo identificou uma maior prevalência de crianças ativas irregulares no grupo estudado (Tabela 5).

Em Belo Horizonte foi estudado o nível de atividade física e o padrão alimentar de 1450 escolares de 6 a 18 anos. Foi demonstrado que $50 \%$ da população estudada apresentou um nível de atividade física menor que o recomendado para uma boa saúde. Em relação ao padrão alimentar, $65 \%$ dos escolares possuíam hábitos muito inadequados e $35 \%$ hábitos inadequados. Foi observada uma prevalência de $8,4 \%$ de sobrepeso e 3,1\% de obesidade (RIBEIRO et al., 2006).

Um grande número de crianças pratica exercícios físicos de forma rotineira apenas na escola, reforçando a importância do currículo escolar incluir aulas de educação física em frequência apropriada (COSTA et al., 2011). Sobretudo em populações carentes, onde essa é a principal forma de prática regular de exercícios físicos. Tal fato foi destacado em outras publicações (KUMANYICA e GRIER, 2006; AHA et al., 2006).

Em Bogotá (Colômbia) foi encontrada uma prevalência de 90,9\% de sedentarismo em crianças com sobrepeso e obesidade. No mesmo estudo, $84,8 \%$ daqueles com sobrepeso e obesidade tinham maus hábitos alimentares (BRICEÑO et al., 2015).

As porcentagens de excesso de peso, incluindo sobrepeso e obesidade, encontradas na literatura, variou entre 5,9\% e 45\%, com uma maior prevalência de eutrofismo em todos eles (BRICEÑO et al., 2015; PEREIRA et al., 2012; MELO et al., 2012; CARVALHO et al., 2014; RIBEIRO et al., 2006). 


\section{CONCLUSÃO}

A prevalência de obesidade infantil no ambulatório de Pediatria do CEMEC, no período compreendido entre setembro a dezembro de 2015 foi de $16 \%$ e $38 \%$ de sobrepeso. Neste estudo não foi possível correlacionar obesidade infantil com obesidade dos pais, fato que pode ser justificado pela amostra utilizada, sendo necessário mais estudos com amostras mais amplas devido à elevada prevalência da obesidade na faixa etária do estudo. A HAS se configurou como um fator de risco relacionado à obesidade. Níveis pressóricos elevados estão relacionados à obesidade em $68,5 \%$ dos escolares com sobrepeso e $100 \%$ dos considerados com obesidade. Os achados do presente estudo não permitiram o estabelecimento de associações entre nível de atividade física e obesidade nos escolares. As modificações do estilo de vida para prevenção e tratamento da obesidade são de fundamental importância para prevenir eventos cardiovasculares no futuro.

\section{REFERÊNCIAS}

1. GIDDING SS, DENNISON BA, BIRCH LL, et al. Dietary recommendations for children and adolescents: a guide for practitioners. Pediatric, 2006 Feb; 117(2): 544-59.

2. BARBOSA E SILVA O. Questionário de avaliação da atividade física e do sedentarismo em criança e adolescente. Revista do DERC, 2009; 15(45): 14-18.

3. BOOTH M, OKELY, A, CHEY T et al. The reliability and validity of the physical activity questions in the WHO health behaviour in schoolchildren (HBSC) survey: a population study. Bristish Journal of Sports Medicine, 2001; 35(4): 263-267

4. Borges ECC, Silva SA. Estado nutricional e hipertensão infantil. Nutrire: rev. Soc. Bras. Alim. Nutr. J. Brazilian Soc. Food Nutr., 2012; 37(3): 259-268.

5. Brasil. Conselho Nacional de Saúde. Resolução $n^{\circ} 466$, de 12 de dezembro de 2012. Diário Oficial da União, $n^{\circ}$ 12, 13 jun 2013, p. 59. Seção 1.

6. Brasil. Instituto Brasileiro de Geografia e Estatística (IBGE). Pesquisa de orçamentos familiares 2008-2009: antropometria e estado nutricional de crianças, adolescente e adultos. 2010.

7. Brasil. Ministério da saúde. Secretaria de Atenção à saúde. Departamento de atenção Básica. Obesidade. Brasília: Ministério da Saúde, 2006. (Caderno de atenção básica, n. 12).

8. BRICEÑO G, FERNÁNDEZ M, CÉSPEDES J. Prevalencia elevada de riesgo cardiovascular en niños. Biomédica 2015; 35:219-26.

9. BURGOS MS, REUTER CP, BURGOS LT et al. Uma análise entre índices pressóricos, obesidade e capacidade cardiorrespiratória em escolares. Arq Bras Cardiol., 2010; 94(6): 739-47

10. COSTA MB, SILVA JHA, SIMÕES ACSR et al. Obesidade infantil: características em uma população atendida pelo programa de saúde da família. Rev APS., 2011; 14(3): 283-288.

11. CARDOSO AS, CARDOSO RO, CARVALHO DF et al. Creative protein and cardiometabolic risk in overweight or obese children and sdolescents. Rev. Nutr., 2014, 27(3).

12. CARVALHO AT, ALMEIDA ER, NILSON EAF et al. Nutritional situation of children under five years old in brazil's northeastern cities. Journal of Human Growth and Development, 2014; 24(2): 221-227.

13. Centers for Disease Control and Prevention, National Center for Health Statistics. 2000 CDC growth charts: United States.

14. COOK S, WEITZMAN M, AUINGER $P$ et al. 2003. Prevalence of a metabolic syndrome phenotype in adolescents: findings from the Third National Health and Nutrition Examinations survey. Arch Pediatr Adolesc Med, 157: 821827.

15. COSTA MB, SILVA JHA, SIMÕES ACSR et al. Obesidade infantil: características em uma população atendida pelo programa de saúde da família. Rev APS., 2011 jul/ set; 14(3): 283-288.

16. FRIEDEMANN C, HENEGHAN C, MAHTANI $\mathrm{K}$ et al. Cardiovascular disease risk in healthy children and its association with body mass index: systematic review and meta-analysis. BMJ, 2012; 345:475.

17. GIBSON LY, BYRNE SM, DAVIS EA et al. The role of family and maternal factors in childhood obesity. Med J Aust 2007; 186:591-595.

18. GIUGLIANO R, CARNEIRO EC. Fatores associados à obesidade em escolares. J Pediatr., 2004; 80(1): 17-22.

19. HINNIG PF. Construção de questionário de frequência alimentar para crianças de 7 a 10 anos. Ver. Bras. Epidemiol. 2014 jun.; 17(2). 
20. KINRA S, NELDER RP, LEWENDON GJ. Deprivation and childhood obesity: a cross sectional study of 20.973 children in Plymouth, United Kingdom. J Epidemiol Comm Health, 2000; 54(6): 456-60.

21. KUMANYIKA S, GRIER S. Targeting interventions for ethnic minority and low-income populations. Fut Child., 2006; 16(1): 187-207.

22. LAKE JK, POWER C, COLE TJ. Child to adult body mass index in the 1958 British birth cohort: associations with parental obesity. Arch Dis Child, 1997; 77:376-381.

23. MELO FM, GONTIJO PDS, BRAGA FA. Obesidade infantil em crianças da rede pública de ensino: prevalência e consequências para flexibilidade, força explosiva e velocidade. Rev. educ. fis. UEM., 2012; 23(4): 629-634.

24. MORAES LI, NICOLA TC, JESUS JSA et al. Pressão arterial elevada em crianças e sua correlação com três definições de obesidade infantil. Arq. Bras. Cardiol., 2014; 102(2).

25. National High Blood Pressure Education Program Working Group on High Blood Pressure in Children and Adolescents. The fourth report on the diagnosis, evaluation, and treatment of high blood pressure in children and adolescents. Pediatrics, 2004; 114 (Suppl 4th Report):555-76.

26. OLIVEIRA AMA, CERQUEIRA EMM, SOUZA JS et al. Sobrepeso e obesidade infantil: influência de fatores biológicos e ambientais em Feira de Santana, BA. Arq Bras Endocrinol Metab. 2003; 47(2): 144-150.

27. PASCHOAL MA, TREVIZAN PF, SCODELER NF. Variabilidade da frequência cardíaca, lípides e capacidade física de crianças obesas e não-obesas. Arq. Bras. Cardiol. 2009, 93(3).

28. PEREIRA A, GUEDES AD, VERRESCHI IT et al. A obesidade e sua associação com os demais fatores de risco cardiovascular em escolares de Itapetininga, Brasil. Arq Bras Cardiol., 2009; 93(3): 253-60.

29. PEREIRA LL, FURLANETTO C, FERREIRA LM et al. Prevalência de sobrepeso e obesidade infantil entre lactentes, pré-escolares e escolares em uma área de abrangência do PET-SAÚDE. Arq. Catarin. Med., 2012; 41(4): 09-14.

30. REIS CE, VASCONCELOS IA, BARROS JFN. Políticas públicas de nutrição para controle da obesidade infantil. Rev. Paul. Pediatr. 2011; 29(4): 626-33.

31. RIBEIRO RQC, LOTUFO PA, LAMOUNIER JA et al. Fatores adicionais de risco cardiovascular associados ao excesso de peso em crianças e adolescentes. O estudo do coração de Belo Horizonte. Arquivos Brasileiros de Cardiologia, 2006; 86(6): 408-18.

32. ROBINSON SM, CROZIER SR, HARVEY NC et al. Modifiable early-life risk factors for childhood adiposity and overweight: an analysis of their combined impact and potential for prevention. Am J Clin Nutr. 2015, 101: 368-75.

33. SILVA MA, RIVERA IR, DE SOUZA MG et al. Blood pressure measurement in children and adolescents: guidelines of high blood pressure recommendations and current clinical practice. Arq Bras Cardiol. 2007; 88(4): 491-5.

34. Sociedade Brasileira de Pediatria. Departamento de Nutrologia, Manual de orientação para a alimentação do lactente, do pré-escolar, do escolar, do adolescente e na escola. 3. ed. Rio de Janeiro: SBP, 2012.

35. TENÓRIO AS, COBAYASHI F. Obesidade infantil na percepção dos pais. Rev. Paul. Pediatr. 2011, 29(4): 6349.

36. World Health Organization. Growth reference 5-19 years.

37. World Health Organization. Obesity: preventing and managing the global epidemic. Report of a World Health Organization Consultation. Genova: World Health Organization, 2000. p.256. WHO Obesity Technical Report Series, n.284. 